\title{
Effects of Neutron-Induced Well Potential Perturbation for Multiple Cell Upset of Flip-Flops in $65 \mathrm{~nm}$
}

\section{$\operatorname{AUTHOR}(\mathrm{S})$ :}

Furuta, Jun; Yamamoto, Ryosuke; Kobayashi, Kazutoshi; Onodera, Hidetoshi

\section{CITATION:}

Furuta, Jun ... [et al]. Effects of Neutron-Induced Well Potential Perturbation for Multiple Cell Upset of Flip-Flops in 65 nm. IEEE Transactions on Nuclear Science 2013, 60(1): 213218

\section{ISSUE DATE:}

2013-01

URL:

http://hdl.handle.net/2433/171285

\section{RIGHT:}

(C)2013 IEEE. Personal use of this material is permitted. Permission from IEEE must be obtained for all other uses, in any current or future media, including reprinting/republishing this material for advertising or promotional purposes,

creating new collective works, for resale or redistribution to servers or lists, or reuse of any copyrighted component of this work in other works.; This is not the published version. Please cite only the published version.; この論文は出版社版

でありません。引用の際には出版社版をご確認ご利用ください。 


\title{
Effects of Neutron-Induced Well Potential Perturbation for Multiple Cell Upset of Flip-Flops in $65 \mathrm{~nm}$
}

\author{
Jun Furuta, Student Member, IEEE, Ryosuke Yamamoto, Kazutoshi Kobayashi, Member, IEEE, \\ and Hidetoshi Onodera, Member, IEEE
}

\begin{abstract}
We measure and investigate the relationship between well potential perturbation and MCUs (Multiple Cell Upsets) by neutron irradiation. Area-efficient cell-based perturbation detectors are placed adjacent to FFs (Flip-Flops). They can measure duration time of perturbation with $5 \mu \mathrm{m}$ spatial resolution at two voltage levels. The measurement results by neutron irradiation on a $65-\mathrm{nm}$ bulk CMOS show that $95 \%$ of MCUs occur simultaneously with well-potential perturbation, while there is very weak relationship between SEUs (Single Event Upsets) and the perturbation.
\end{abstract}

Index Terms-Multiple Cell Upset, soft-error, neutron irradiation, sensor, parasitic bipolar effect.

\section{Introduction}

As a result of process scaling, MCU is becoming one of the most significant issues for LSI (Large Scale Integration) reliability since it can flip several stored values on SRAMs (Static Random Access Memories) and cannot be recovered by ECC (Error Correction Code) [1]. In a $65-\mathrm{nm}$ process, MCUs are observed in FFs and increase soft-error rates of radiation-hardened FFs such as multiple modular redundancy [2], [3]. SEMTs (Single Event Multiple Transients) are also reported by neutron and heavy-ion experiments [4], [5]. In a future advanced process, it is impossible to improve softerror resilience of flip-flops without considering such multiple errors.

The parasitic bipolar effect is considered as a source of multiple errors. It is caused by well-potential perturbation due to a particle hit [6], [7]. Since well-potential perturbation is strongly affected by well-contact density [8], MCU rates also depend on the distance from well-contacts [9], the angle of particle [10], [11] and back bias [6]. Ref. [12] also shows parasitic bipolar effect increases widths of SET (Single Event Transient) pulses. Parasitic bipolar and well-potential perturbation are significant phenomena that cause single and multiple errors. For estimating soft error rate from circuit-level simulations and improving soft-error resilience, it is necessary to measure well-potential perturbation caused by a particle hit and evaluate relationship between well-potential perturbation

J. Furuta and H. Onodera are with Graduate School of Informatics, Kyoto University (e-mail: furuta@vlsi.kuee.kyoto-u.ac.jp; onodera@vlsi.kuee.kyotou.ac.jp).

R. Yamamoto and K. Kobayashi are with Graduate School of Science \& Technology, Kyoto Institute of Technology (e-mail: kazutoshi.kobayashi@kit.ac.jp and soft errors. However, no one shows measurement results of well-potential perturbation caused by a particle hit. Only simulation results are reported in [7], [8], [11].

In this paper, we show actual measurement results of well-potential perturbation by neutron irradiation using wellpotential perturbation detectors based on [13]. We also show the relationship between well-potential perturbations and MCU occurrences on FFs. Test structures are fabricated in a 65-nm bulk CMOS process and they can measure duration time of perturbation with $5 \mu \mathrm{m}$ spatial resolution at two voltage levels. Accelerated tests were carried out by the spallation neutron beam at RCNP (Research Center for Nuclear Physics, Osaka University).

This paper is organized as follows. Section II explains the test circuit structure in detail. Section III shows our neutronbeam experimental setups in RCNP, followed by Section IV which discusses experimental results. Section V concludes this paper.

\section{Test Circuit Structure}

Fig. 1 shows unit circuits of the well-potential perturbation detector [13]. They consist of AND / OR chains and two time-to-digital converters (TDCs) based on the ring oscillator [14]. An input port of each logic gate is connected to the Pwell or N-well through an inverter. If a particle hit on a chip and well-potential is elevated over the threshold voltage, the inverter is flipped until well-potential goes back to less than the threshold voltage. Therefore, well-potential perturbation is converted to a rectangular pulse by the inverter and it propagates through the logic chain. Finally, the TDC detects well-potential perturbation and measures its duration. The measurable voltage level of the proposed circuit is determined by the threshold voltage of the inverter, which can be easily changed by skewing transistor sizes of inverters.

Fig. 2 shows a block diagram of the fabricated test circuit. The purposes of this test circuit are as follows.

- To measure the relationship between well-potential perturbation and MCUs on FFs.

- To reveal that SEU on FFs can be detected by measuring well-potential perturbation. If SEU is strongly correlated, it can be detected by proposed circuit and built-in current sensors [15].

- To obtain the characteristics of well-potential perturbation caused by a neutron strike such as dependence of the well-contact distance. 


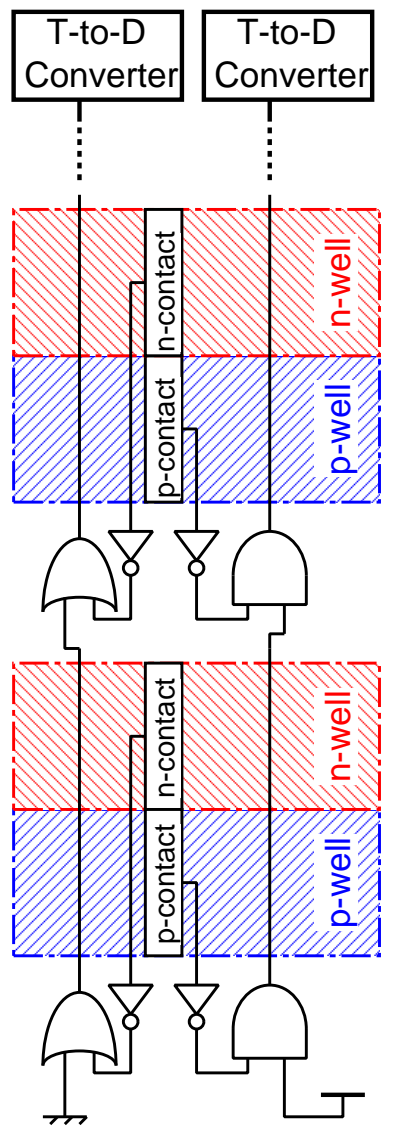

Fig. 1. Well-Potential perturbation detector composed of AND chain, OR chain and two T-to-D converters.

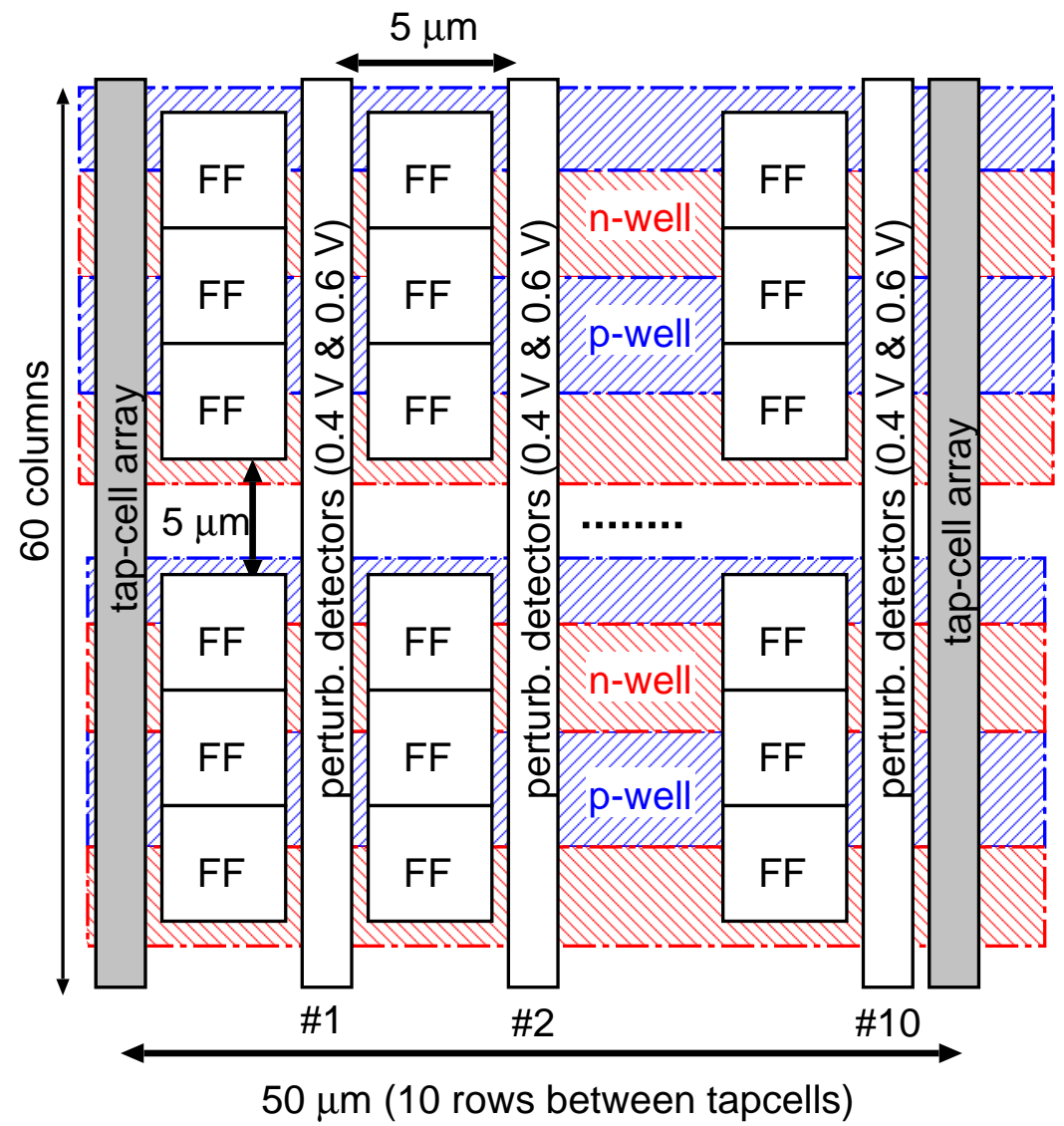

Fig. 2. Block diagram of measurement circuit with detector and shift register.
As shown in Fig. 2, FF arrays and two types of detectors are implemented every $5 \mu \mathrm{m}$. FFs arrays are composed of a $60 \times 100$-bit shift register. Fig. 3 shows the schematic diagram of the implemented FF. Tap-cell arrays are inserted into every $50 \mu \mathrm{m}$. The tap-cell is constructed by $\mathrm{N}$-well and $\mathrm{P}$-well contacts and it stabilizes well-potential. There are 10 columns of FF arrays and detectors between two tap-cell columns. The threshold voltages of detectors are $0.4 \mathrm{~V}$ and $0.6 \mathrm{~V}$. The latter threshold voltage, $0.6 \mathrm{~V}$ is equal to the threshold voltage value above which the current flows to PN diode between well and drain regions. We assume that if an MCU is caused by the parasitic bipolar effect, the proposed circuit detects $0.6 \mathrm{~V}$ perturbation of well-potential whenever an MCU occurs on FFs. The $0.4 \mathrm{~V}$ perturbation detector is fabricated to detect SEUs and also to duplicate perturbation detector and remove wrong detections by an SEU on $0.6 \mathrm{~V}$ perturbation detector.

In this test circuit, duration time of well-potential perturbations is measured with $5 \mu \mathrm{m}$ spatial resolution at two voltage levels, $0.4 \mathrm{~V}$ and $0.6 \mathrm{~V}$. In order to remove the parasitic bipolar effect on the chains caused by well-potential perturbation, they are placed on wells isolated from the measured ones.

\section{Experimental Setup}

Fig. 4 shows a test chip micrograph fabricated in a 65$\mathrm{nm}$ bulk CMOS process. It is implemented by tapless standard cells [16] on a twin-well (dual-well) structure. In order to measure neutron-induced well-potential perturbation and MCU rates on FFs, a $6,000(60 \times 100)$ bit shift register is

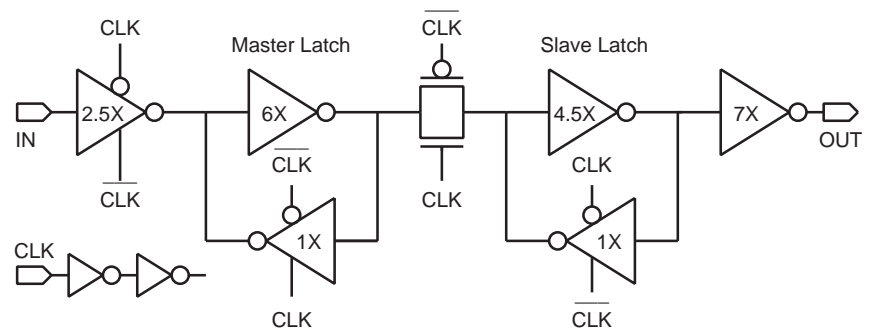

Fig. 3. Schematic diagram of implemented FF.

implemented and its total area is $0.5 \times 0.6 \mathrm{~mm}^{2}$ on a $2 \times 4$ $\mathrm{mm}^{2}$ die.

Accelerated tests were carried out at RCNP (Research Center for Nuclear Physics, Osaka University). Fig. 5 shows the neutron beam spectrum compared with the terrestrial neutron spectrum at the ground level of Tokyo. The average acceleration factor is $3.8 \times 10^{8}$. In order to increase error counts, we measured 28 chips at the same time using stacked DUT (Device Under Test) boards. An engineering LSI tester is used to control DUTs and collect shifted error data. During irradiation, stored values of the shift register are initialized to "ALL0" and clock signal is fixed to "1" to keep master latches on FFs in the hold state. Stored values are retrieved every 5 minutes. 


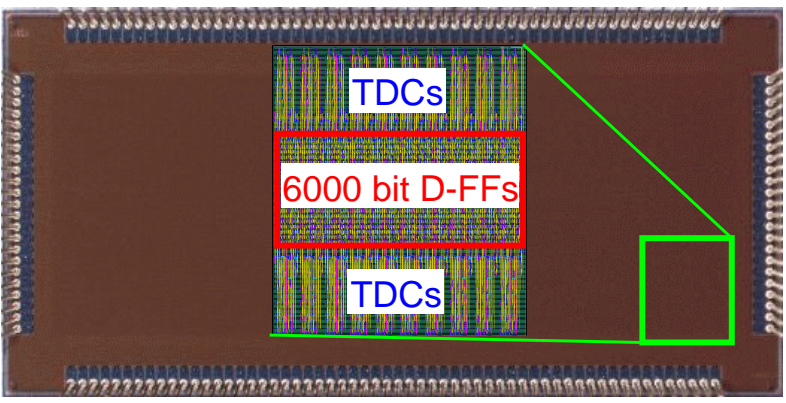

Fig. 4. Chip micrograph and floorplan.

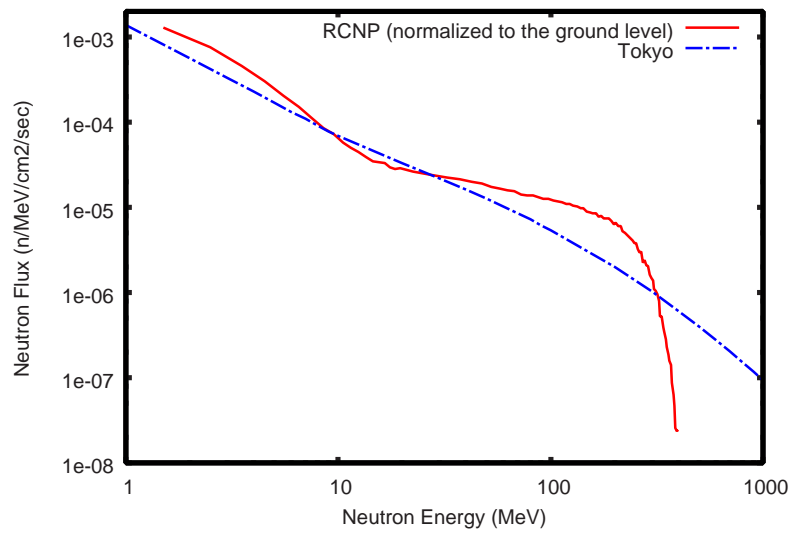

Fig. 5. Neutron spectrum at RCNP.

\section{Experimental Results and Discussions}

In this section, we show measurement results of wellpotential perturbation and soft errors on FFs. To show measurement results shortly, we use these abbreviations as shown in Table I.

\section{A. Relationship between Well-Potential Perturbations and Soft-Error on FFs}

Table II shows the total number of SEUs, MCUs and WPPs. $M C U_{\text {vertical }}$ shows the number of flips on 2 bit FFs between vertically-contacted cells, whose master latches are separated by $0.7 \mu \mathrm{m}$. $\mathrm{MCU}_{\text {horizontal }}$ shows the number of flips on $2 \mathrm{bit}$ FFs between horizontally-contacted cells, whose master latches are separated by $5 \mu \mathrm{m}$. The rates of WPPs are about $1-4 \mathrm{x}$ bigger than the SEU rate on FFs. It is because well-potential perturbation may happen without SEUs when a particle hits on the circuit blocks which do not cause an SEU such as clock buffers or slave latches in the transparent state.

Fig. 6 shows the relationship between well-potential perturbation and soft-errors on FFs. The relationship between

TABLE I

LIST OF ABBREVIATION.

\begin{tabular}{|l||c|}
\hline Abbreviation & Meaning \\
\hline \hline WPP & well-potential perturbation \\
\hline WPP[P, 0.4 V] & P-well-potential perturbation over $0.4 \mathrm{~V}$ \\
\hline WPP[P, 0.6 V] & P-well-potential perturbation over $0.6 \mathrm{~V}$ \\
\hline WPP[N, 0.4 V] & N-well-potential perturbation over $0.4 \mathrm{~V}$ \\
\hline WPP[N, 0.6 V] & N-well-potential perturbation over $0.6 \mathrm{~V}$ \\
\hline
\end{tabular}

TABLE II

THE TOTAL NUMBER OF WPPS, SEUS AND MCUS ON FFS.

\begin{tabular}{|l||r|}
\hline & Count \\
\hline \hline SEU & 455 \\
\hline MCU $_{\text {vertical }}$ & 33 \\
\hline MCU $_{\text {horizontal }}$ & 4 \\
\hline WPP[P, 0.4 V] & 1514 \\
\hline WPP[P, 0.6 V] & 709 \\
\hline WPP[N, 0.4 V] & 1633 \\
\hline WPP[N, 0.6 V] & 565 \\
\hline
\end{tabular}

TABLE III

AVERAGE DURATION TIME OF WPPS WHEN SOFT-ERROR ON FF OCCURS WITH WPP.

\begin{tabular}{|l||r|r|r|r|}
\hline \multicolumn{1}{|c||}{} & \multicolumn{4}{c|}{ Average duration time of WPP } \\
\cline { 2 - 5 } & {$[\mathrm{P}, 0.4 \mathrm{~V}]$} & {$[\mathrm{P}, 0.6 \mathrm{~V}]$} & {$[\mathrm{N}, 0.4 \mathrm{~V}]$} & {$[\mathrm{N}, 0.6 \mathrm{~V}]$} \\
\hline \hline No error & $530 \mathrm{ps}$ & $350 \mathrm{ps}$ & $670 \mathrm{ps}$ & $460 \mathrm{ps}$ \\
\hline SEU & $930 \mathrm{ps}$ & $750 \mathrm{ps}$ & $920 \mathrm{ps}$ & $880 \mathrm{ps}$ \\
\hline $\mathrm{MCU}_{\text {vertical }}$ & $1070 \mathrm{ps}$ & $800 \mathrm{ps}$ & $1030 \mathrm{ps}$ & $920 \mathrm{ps}$ \\
\hline MCU $_{\text {horizontal }}$ & $1450 \mathrm{ps}$ & $1200 \mathrm{ps}$ & $1400 \mathrm{ps}$ & $1220 \mathrm{ps}$ \\
\hline
\end{tabular}

WPPs and MCUs are very strong and 95\% (35 / 37) of MCUs occur with WPP[P, 0.6 V]. However, no MCU occurs with WPP $[\mathrm{P}, 0.4 \mathrm{~V}]$ but without WPP[P, $0.6 \mathrm{~V}]$ as shown in Fig. 6. Therefore, MCUs occur on FFs when the voltage level of P-well-potential perturbation is under $0.4 \mathrm{~V}$ or over $0.6 \mathrm{~V}$. We conclude that all MCUs are not caused by the identical mechanism. All MCUs except for two are caused by the parasitic bipolar effect and two MCUs are caused by different mechanisms such as charge sharing or successive hits by one ion [3]. In the fabricated technology, the parasitic bipolar effect is a critical issue for soft-error resilience on redundant flip-flops.

As shown in Fig. 6 and our previous work [13], WPP is weakly overlapped with SEUs on FFs. The WPP detector or bulk built-in current sensor cannot detect all SEU on FFs. However, it can be used as an error detector for redundant FFs because WPP is almost overlapped with MCUs on FFs. The proposed detector and a roll-back function increase softerror resilience of a radiation-hard system using redundant FFs by 20 times since $95 \%$ ( $35 / 37$ ) of MCUs can be detected by the proposed detector.

Table III shows the average duration time of WPPs when a soft error on FF occurs with WPP. When MCU occurs on FFs, well-potential is perturbed for about 1000 ps at $0.4 \mathrm{~V}$. Especially in the case of horizontal MCU occurrence, it is perturbed for 1,400 ps at $0.4 \mathrm{~V}$ and for 1,200 ps at $0.6 \mathrm{~V}$.

\section{B. Characterization of Well-Potential Perturbations}

Here, we show measurement results of WPPs. Fig. 7 shows an example of measurement results observing an MCU detected by wide-spread WPPs. The test circuit can detect WPP properly since WPP $[\mathrm{P}, 0.4 \mathrm{~V}]$ (WPP[N, $0.4 \mathrm{~V}])$ always detect longer WPP than WPP $[\mathrm{P}, 0.4 \mathrm{~V}](\mathrm{WPP}[\mathrm{N}, 0.6 \mathrm{~V}])$ and longest durations of WPPs are detected at the same location, \#4. Wellpotential is perturbed for $400-600$ ps at $0.6 \mathrm{~V}$.

Fig. 8 and 9 show the number of well-potential perturbations by keeping clock signal to " 1 " during irradiation. X-axis shows the number of the WPP detectors which detect WPP 


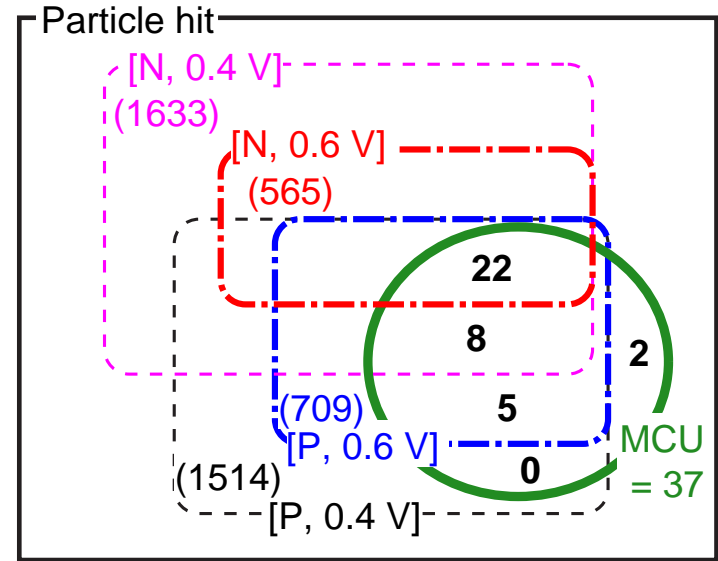

$\mathrm{MCU}$

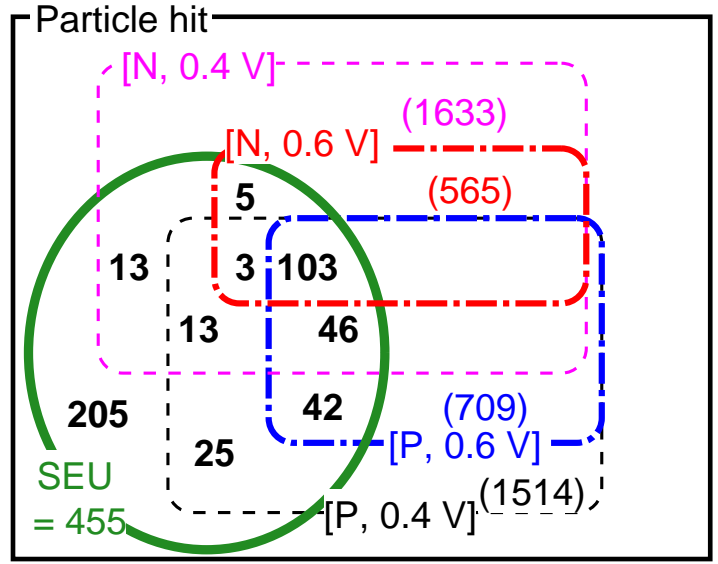

SEU

Fig. 6. Venn diagrams showing the relationship between WPPs and MCUs and SEUs. Values in parentheses show the total number of WPP.

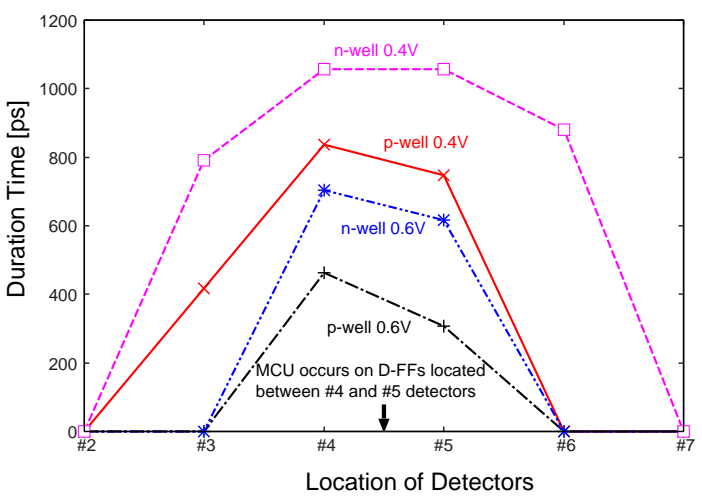

Fig. 7. Example of measurement results for well-potential perturbation with MCU occurrence on FFs placed between \#4 and \#5 detectors.

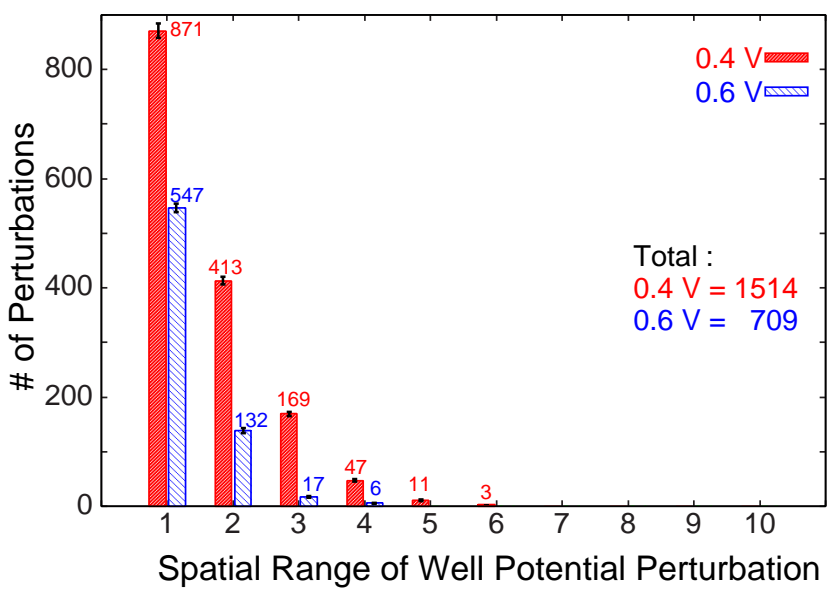

Fig. 8. Spatial range of P-well-potential perturbations.

simultaneously. It is equal to spatial range of WPP since the WPP detectors are implemented every $5 \mu \mathrm{m}$ as shown Fig. 2 . Comparing WPPs in P-well and in N-well, the number of WPP $[N, 0.4 \mathrm{~V}]$ is larger than that of WPP $[\mathrm{P}, 0.4 \mathrm{~V}]$. This is because the transistor width and drain area of pMOS are larger than that of nMOS. However, the number of WPPs $[\mathrm{N}$, $0.6 \mathrm{~V}]$ is smaller than those of WPPs[P, $0.6 \mathrm{~V}]$. We assume that electrons generated by a neutron strike in $\mathrm{N}$-well are diffused

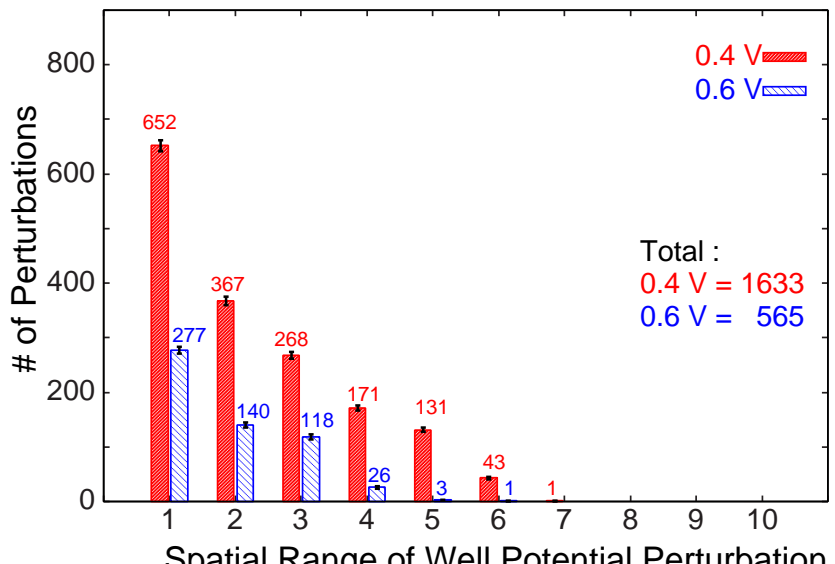

Fig. 9. Spatial range of N-well-potential perturbations.

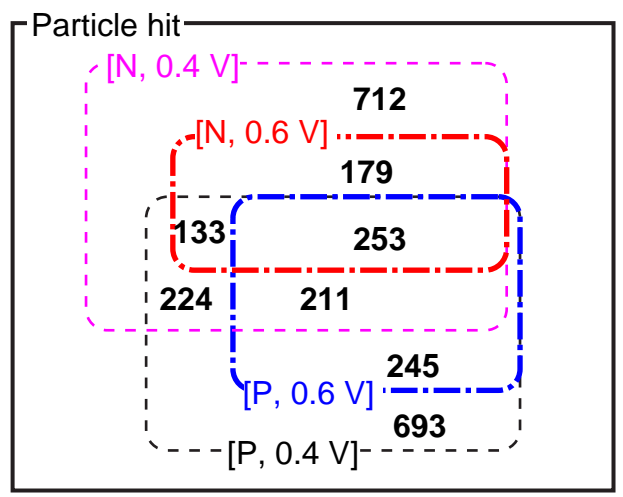

Fig. 10. Venn diagram showing the relationship among WPPs.

more quickly and N-well-potential is not easily reduced since the electron mobility is bigger than the hole mobility. The spatial range of $\mathrm{N}$-well-potential perturbations is wider than that of P-well-potential perturbations. The difference of the spatial ranges is also caused by the difference of $\mathrm{N}$-well and $\mathrm{P}$-well structures. Our test chip is implemented with the twinwell structure. The depth of $\mathrm{N}$-well is much thinner than $\mathrm{P}$ well (P-substrate).

Fig. 10 shows relationship between P-well-potential and N- 


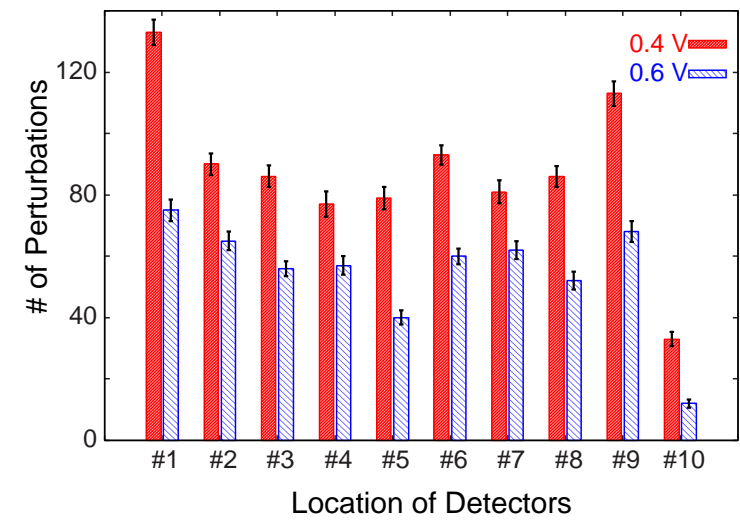

Fig. 11. The number of P-well-potential perturbation detected by one detector according to the distance from tap-cells.

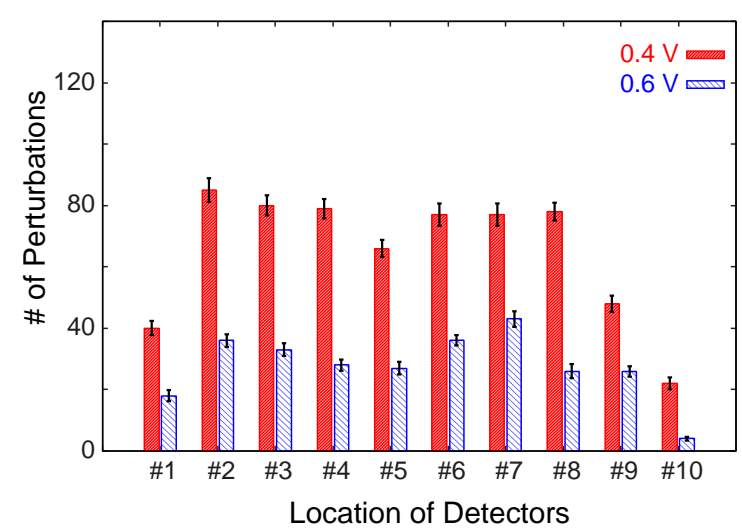

Fig. 13. The number of N-well-potential perturbation detected by one detector according to the distance from tap-cells.

well-potential perturbations. About a half of WPPs[N, 0.4 V] occurs simultaneously with WPP $[\mathrm{P}, 0.4 \mathrm{~V}]$. We assume that it is caused by the diffusion current flowing into a PN junction between P-well and N-well.

Fig. 11-14 show the number of well-potential perturbations detected by one or three adjacent detectors respectively according to the distance from tap-cells. As shown in Fig. 13 and 14, the number of N-well-potential perturbations depends on the distance from tap-cells. The number of WPP[N, 0.4 V] detected by \#1, \#9 and \#10 detectors is smaller than that detected by \#2 - \#8 detectors which are separated from tapcell by over $10 \mu \mathrm{m}$. In contrast, the number of P-well-potential perturbations does not depend on the distance from tap-cells. The \#1 detector detects the maximum number of WPP[P, 0.4 V].

The number of P-well-potential perturbation is small detected by the \#10 detector which is nearest to tap-cells. Therefore, even if tap-cells are inserted every $5 \mu \mathrm{m}$, MCU rate on FFs is not reduced drastically. In order to reduce WPPs and MCU rate on FFs, we should not fabricate a chip composed of tapless standard cells. It must be composed of conventional standard cells which have well-contacts under their power and ground rails.

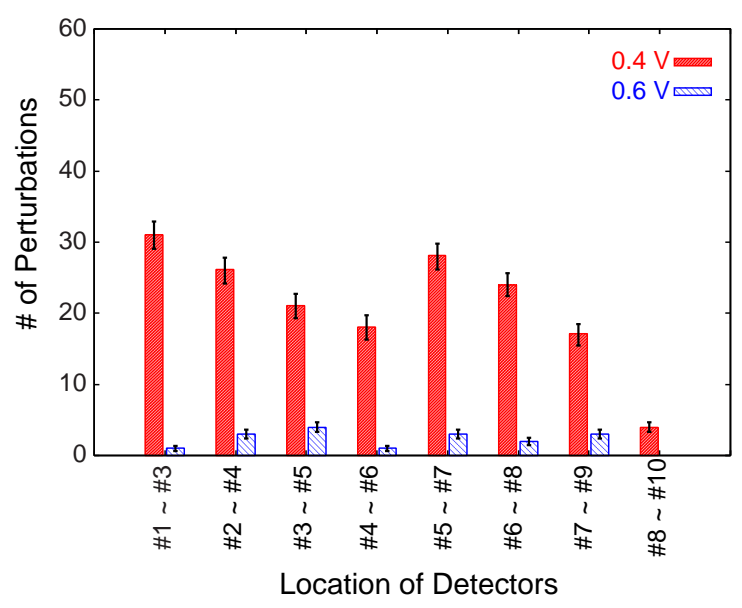

Fig. 12. The number of P-well-potential perturbation detected by three adjacent detectors according to the distance from tap-cells.

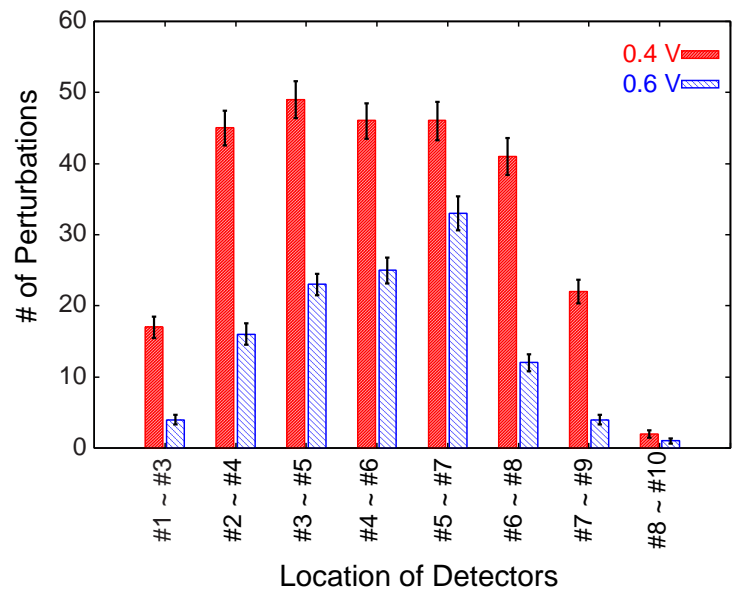

Fig. 14. The number of N-well-potential perturbation detected by three adjacent detectors according to the distance from tap-cells.

\section{Conclusion}

We show measurement results of well potential perturbation caused by a particle hit from spallation neutron irradiation. The implemented detectors can measure duration time of wellpotential perturbation with $5 \mu \mathrm{m}$ spatial resolution at two voltage levels, $0.4 \mathrm{~V}$ and $0.6 \mathrm{~V}$. Experimental results by neutron irradiation on a $65-\mathrm{nm}$ bulk CMOS show that rate of well-potential perturbation is 1 to 4 times bigger than the SEU rate on FFs and perturbation durations of over 1,000 ps are also measured. 95\% of MCUs are detected simultaneously with well potential perturbation. There is a very strong overlap between well-potential perturbation and MCUs on FFs. To reduce MCU rates on FFs or SEU rates on redundant FFs, a chip must be composed of conventional standard cells which have well-contacts under their power and ground rails.

\section{ACKNOWLEDGMENT}

The authors would like to thank to Prof. K. Hatanaka, Prof. M. Fukuda, and Prof. K. Takahisa at RCNP and all the other RCNP staffs for our neutron-beam experiments. This work is partly supported by Grant-in-Aid for JSPS Fellows (24.7662). The VLSI chip in this study has been fabricated in the chip fabrication program of VLSI Design and Education 
Center(VDEC), the University of Tokyo in collaboration with STARC, e-Shuttle, Inc., and Fujitsu Ltd.

\section{REFERENCES}

[1] G. Gasiot, P. Roche, and P. Flatresse, "Comparison of multiple cell upset response of bulk and SOI 130nm technologies in the terrestrial environment," in IRPS, May 2008, pp. $192-194$.

[2] R. Yamamoto, C. Hamanaka, J. Furuta, K. Kobayashi, and H. Onodera, "An area-efficient $65 \mathrm{~nm}$ radiation-hard dual-modular flip-flop to avoid multiple cell upsets," IEEE Trans. Nucl. Sci., vol. 58, no. 6, pp. 3053 -3059, Dec. 2011.

[3] T. Uemura, T. Kato, H. Matsuyama, K. Takahisa, M. Fukuda, and K. Hatanaka, "Investigation of multi cell upset in sequential logic and validity of redundancy technique," in IOLTS, July 2011, pp. $7-12$

[4] R. Harada, Y. Mitsuyama, M. Hashimoto, and T. Onoye, "Neutron induced single event multiple transients with voltage scaling and body biasing," in IRPS, Apr. 2011, pp. 3C.4.1 -3C.4.5.

[5] J.R. Ahlbin, T.D. Loveless, D.R. Ball, B.L. Bhuva, A.F. Witulski, L.W. Massengill, and M.J. Gadlage, "Double-pulse-single-event transients in combinational logic," in IRPS, Apr. 2011, pp. 3C.5.1 -3C.5.6.

[6] T. Nakauchi, N. Mikami, A. Oyama, H. Kobayashi, H. Usui, and J. Kase, "A novel technique for mitigating neutron-induced multi-cell upset by means of back bias," in IRPS, May 2008, pp. $187-191$.

[7] J.D. Black, D.R. Ball, W.H. Robinson, D.M. Fleetwood, R.D. Schrimpf, R.A. Reed, D.A. Black, K.M. Warren, A.D. Tipton, P.E. Dodd, N.F. Haddad, M.A. Xapsos, H.S. Kim, and M. Friendlich, "Characterizing SRAM single event upset in terms of single and multiple node charge collection," IEEE Trans. Nucl. Sci., vol. 55, no. 6, pp. 2943 -2947, Dec. 2008.

[8] N.J. Gaspard, A.F. Witulski, N.M. Atkinson, J.R. Ahlbin, W.T. Holman, B.L. Bhuva, T.D. Loveless, and L.W. Massengill, "Impact of well Structure on single-event well potential modulation in bulk CMOS," IEEE Trans. Nucl. Sci., vol. 58, no. 6, pp. 2614 -2620, Dec. 2011.
[9] D. Radaelli, H. Puchner, S. Wong, and S. Daniel, "Investigation of multi-bit upsets in a $150 \mathrm{~nm}$ technology SRAM device," IEEE Trans. Nucl. Sci., vol. 52, no. 6, pp. 2433 - 2437, Dec. 2005.

[10] A.D. Tipton, J.A. Pellish, J.M. Hutson, R. Baumann, X. Deng, A. Marshall, M.A. Xapsos, H.S. Kim, M.R. Friendlich, M.J. Campola, C.M. Seidleck, K.A. LaBel, M.H. Mendenhall, R.A. Reed, R.D. Schrimpf, R.A. Weller, and J.D. Black, "Device-orientation effects on multiple-bit upset in $65 \mathrm{~nm}$ SRAMs," IEEE Trans. Nucl. Sci., vol. 55, no. 6, pp. 2880 -2885, Dec. 2008.

[11] A.D. Tipton, Xiaowei Zhu, Haixiao Weng, J.A. Pellish, P.R. Fleming, R.D. Schrimpf, R.A. Reed, R.A. Weller, and M. Mendenhall, "Increased rate of multiple-bit upset from neutrons at large angles of incidence," IEEE Trans. Device and Materials Reliability, vol. 8, no. 3, pp. 565 -570 , Sept. 2008

[12] B. Narasimham, B.L. Bhuva, R.D. Schrimpf, L.W. Massengill, M.J. Gadlage, T.W. Holman, A.F. Witulski, W.H. Robinson, J.D. Black, J.M. Benedetto, and P.H. Eaton, "Effects of guard bands and well contacts in mitigating long SETs in advanced CMOS processes," IEEE Trans. Nucl. Sci., vol. 55, no. 3, pp. $1708-1713$, June 2008.

[13] J. Furuta, R. Yamamoto, K. Kobayashi, and H. Onodera, "Correlations between well potential and SEUs measured by well-potential perturbation detectors in 65nm," in A-SSCC, Nov. 2011, pp. $209-212$.

[14] J. Furuta, R. Yamamoto, K. Kobayashi, and H. Onodera, "Evaluation of parasitic bipolar effects on neutron-induced SET rates for logic gates," in IRPS, Apr. 2012, pp. SE.5.1 - SE.5.5.

[15] E.H. Neto, I. Ribeiro, M. Vieira, G. Wirth, and F.L. Kastensmidt, "Evaluating fault coverage of bulk built-in current sensor for soft errors in combinational and sequential logic," in VLSI Cir. Symp., Sept. 2005, pp. $62-67$.

[16] S. Idgunji, "Case study of a low power MTCMOS based ARM926 SoC : design, analysis and test challenges," in IEEE Int. Test Conference, Oct. 2007, pp. $1-10$. 